\title{
Feuding hampers Internet's growth in Japan
}

Tokyo. The use of Internet, the global network of computer networks which links an estimated 20 million users worldwide, is about to take off in Japan. Two newly established companies are vying to provide commercial access to the network; and the government is about to upgrade the quality of the public sector portion of the Internet system, made up of computer networks linking universities and national institutes.

But rivalries between those responsible for competing networks in both the public and private sectors are still preventing the cooperation needed to ensure their maximum effectiveness. Such feuding over the control of Japan's computer networks is thus hindering their full development.

Japan has been slow to acknowledge the importance of computer networks, and the first high-capacity links to networks outside Japan were established through the initiatives of individual scientists rather than the government (see Nature 340, 670; 1989 and 356, 550; 1992).

The capacity of these links remains relatively low, and Internet traffic between Japan and the rest of the world is still limited in comparison with other advanced nations, being exceeded even by its small neighbour Taiwan (see caption above). But this situation is likely to change later in the year as the private sector and the government move to improve Japan's overseas computer links.

The first commercial provider of access to Internet has been InterCon International KK, a subsidiary of the US company InterCon Systems Corporation. This now offers full services with e-mail, file transfer and the ability to log into computers around the world. Another Japanese company, Internet Initiative Japan (IIJ), is expected to obtain a licence within the next month or so from the Ministry of Posts and Telecommunications to offer similar services.

But the start of commercial services has not been smooth. One of InterCon's first customers, a small Tokyo-based network called TWICS backed

by a non-profit educational foundation, was abruptly disconnected from its e-mail link to Tokyo University in November by the university's computer centre.

The disconnection took place shortly after TWICS signed up with InterCon, and inconvenienced several hundred TWICS users. TWICS was reconnected only after the intervention of Jun Murai of Keio University, the father of computer networks in Japan, and an adviser to IIJ.

Roger Boisvert, the president of InterCon, claimed that the "old boy network" of Tokyo University, IIJ and the managers of university networks in Japan were taking their revenge on TWICS for choosing InterCon rather than IIJ. The latter company has denied Boisvert's charges, and the university describes the incident as a "misunderstanding" over the timing of TWICS' move to InterCon.

But the affair has drawn attention to the competition that still exists between different networks. This year, the Science and Technology Agency (STA), with the support of various government ministries, will establish a new high-capacity interministerial network linking national institutes.

The network will have a high-capacity link to NSFNET in the United States, and a gateway to the Science Information Network (SINET) that links Japan's universities. It is run by the National Center for

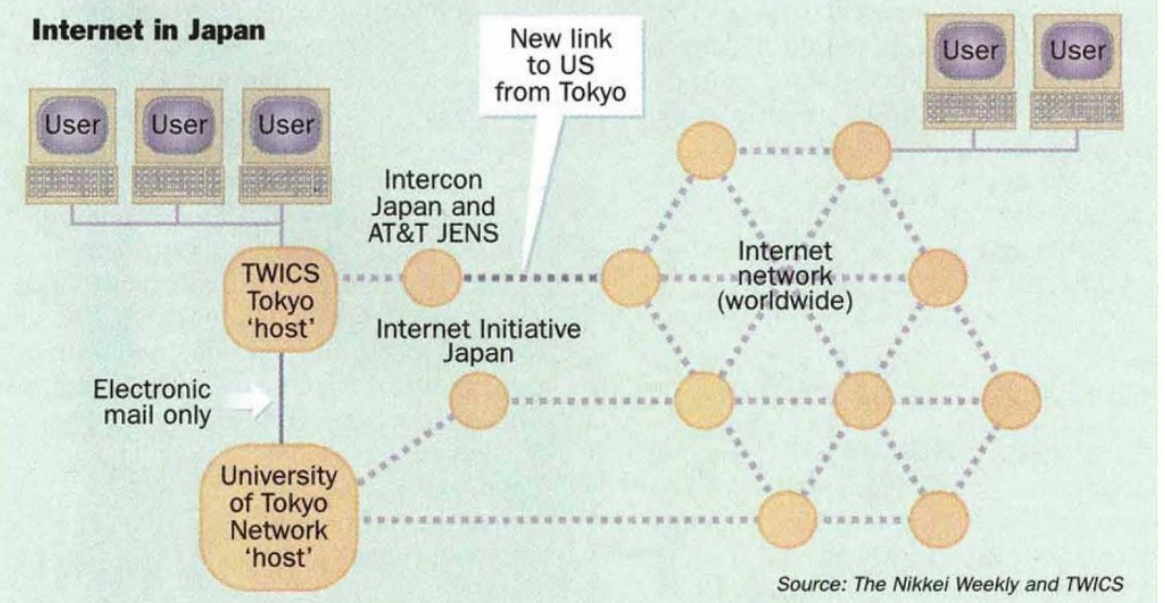
the Ministry of Education, Science and Culture.

But as STA and NACSIS talk publicly of cooperation, each is manoeuvring to gain more control. STA plans to absorb into the new network the Todai (Tokyo University) International Science Network (TISN), established in 1989 on the initiative of individual Tokyo University researchers, which provides a link to the United States used by numerous universities and research institutes. According to one TISN user, however, NACSIS has been quietly lobbying TISN users to join its network.

Tateo Arimoto, director of STA's science and technology information division, hopes that eventually the various government-run networks will be merged into one "seamless" system. That dream still seems a long way off.

At present, at least six governmentrelated networks have links to overseas: TISN, SINET, the Widely Integrated Distributed Environment (WIDE) established by Jun Murai, the Real World Computing project run by the Ministry of International Trade and Industry, STA's Japan Information Centre of Science and Technology, and the Japanese section of the BITNET network, run by Tokyo Science University.

Many observers feel that these organizations should pool their resources and buy one very-high-capacity international link. According to Arimoto, the US National Science Foundation (NSF) has been strongly urging Japan to do just that. But such cooperation still seems a long way off.

David Swinbanks 